\title{
Congenital Extrahepatic Portosystemic Deviation in a Mixed-Breed Dog
}

\author{
Ellen Bethânia de Oliveira Cavalcanti', Gustavo Cancian Baioto'², Clairton Marcolongo-Pereira ${ }^{3}$, \\ Mayara Coutinho Carlos de Souza', Alice Corrêa Rassele' \& Rodrigo dos Santos Horta'
}

\begin{abstract}
Background: Portosystemic deviation (PSD) is a congenital or acquired vascular anomaly that allows an abnormal blood flow from the portal vein directly to the systemic circulation. This liver by-pass avoids hepatic metabolism of several toxins. Congenital PSDs are usually solitary and extra-hepatic, with a high incidence in pure-breed dogs. Acquired PSDs are usually multiple and occur as a consequence of portal hypertension. Surgery is the definitive treatment. Clinicians and surgeons may present difficulties in the propaedeutic of animals with PSD. This paper aims at reporting a successful surgical treatment of a solitary extra-hepatic congenital PSD in a mixed-breed dog.

Case: A 7-month-old mixed-breed dog, female, spayed, weighing $8 \mathrm{~kg}$, was presented with a history of sudden syncope after feeding. Complementary exams revealed normocytic hypochromic anaemia, hypoalbuminemia and increased alanine aminotransferase and alkaline phosphatase. Abdominal ultrasound revealed an anomalous vessel inserted in the caudal cava vein, compatible with a congenital extra-hepatic PSD. Computed tomography revealed the anomalous vessel, with $1,1 \mathrm{~cm}$ of diameter, originated from the cranial mesenteric vein and it inserted in the cranial margin of the caudal cava vein. A medical support was started with hydration, metronidazole, lactulose, probiotic and Hepatic diet. After 15 days the dog was submitted to surgery and a $5 \mathrm{~mm}$ ameroid constrictor ring was placed to gradually close the anomalous vessel. The dog recovered well and an abdominal ultrasound was repeated after 30 days, showing the ameroid constrictor ring ring in the left cranial abdominal region, occluding the PSD close to its insertion in the caudal cava vein. The patient was followed up for more than three years, gained weight and presented a healthy normal life.

Discussion: Although PSD is more often diagnosed in pure-breed dogs, this paper reports a case in a mixed-breed dog. However clinical signs and the age of onset were compatible with this pathology. Dogs with PSD may present neurologi$\mathrm{cal}$, gastrointestinal and urinary disorders, related to failure of the detoxification process and toxin by-pass to the systemic circulation. Young dogs are usually presented overdue underdevelopment, reduced body height or weight loss as described in this report. Hepatic encephalopathy is a result of the production of ammonia and several other toxins, which escapes hepatic metabolism, and once they remain liposoluble, they are able to cross-pass the blood-brain barrier and produce the neurological signs, which might be more pronounced after feeding, as in the reported case, although this dog presented only mild neurological signs. The haematological and biochemical findings in the dog of this report were also compatible with PSD. Hypoglycemia, hypocholesterolemia, increased of bile acids and serum ammonia may also be present but they were not demonstrated in this case. Abdominal ultrasound was able to identify the anomalous extra-hepatic vessel which was precisely described by computed tomography, which allowed the surgical planning. Medical support is recommended for the patient's clinical estabilization, as performed in this case. Surgery is the treatment of choice for congenital solitary PSD, as in the reported case, and placement of the ameroid constrictor is the most employed technique, as performed. Patient's improvement starts in the day after surgery and it is completed within a couple months. The patient of this report did not present any complications in the postoperative period and for more than three years, showing total remission of the clinical signs and good life's quality.
\end{abstract}

Keywords: surgery, portosystemic shunt, ameroid ring, mixed-breed dog. 


\section{INTRODUCTION}

Portosystemic deviation (PSD) or portosystemic shunt is a vascular anomaly that allows an abnormal blood flow from the portal vein directly into the systemic circulation. This results in systemic persistence of toxins from the intestines that would normally be removed and metabolized by the liver, which might result in systemic effects $[2,10,11]$. In addition, important hepatotropic substances, do not reach the liver, resulting in its atrophy [10]. PSD has been described in several species and may be congenital or acquired, intra- or extra-hepatic, solitary or multiple $[1,3]$.

Congenital PSDs are equivalent to $80 \%$ of cases, and most of them are formed by solitary extra-hepatic anomalous vessels [2,3]. Congenital extrahepatic PSDs represent $63 \%$ of solitary deviations in dogs and a higher incidence is reported in pure-breed dogs, especially Yorkshire Terrier [15]. Congenital intra-hepatic deviations occur more frequently in dogs of large and giant breeds, and the solitary presentation is also more common [8,11]. Acquired PSD differs from congenital because it occurs due to an organic compensation in situations of portal hypertension [3]. The acquired deviations are usually multiple [9]. The definitive treatment for PSD is surgical, but only patients with congenital PSD are candidates for surgery, overdue the high risk of portal hypertension and complications in the acquired PSD $[9,11]$.

This paper aims at reporting a successful surgical treatment of a solitary extra-hepatic congenital PSD in a mixed-breed dog, as an example to guide clinicians through diagnosis and treatment of this condition.

\section{CASE}

A 7-month-old mixed-breed dog, female, spayed, weighing $8 \mathrm{~kg}$, was presented with a history of syncope, evidenced immediately after feeding. It has also been reported underdevelopment compared to the rest of the litter. Physical examination showed hypochromic mucosa, tachycardia, tachypnea and rectal temperature of $37.5^{\circ} \mathrm{C}$. The patient was admitted for observation and complementary tests.

Complete blood count showed normocytic hypochromic anaemia and mild neutrophilic leukocytosis with left shift. Serum biochemistry evidenced hypoalbuminemia, increase of alanine aminotransferase and alkaline phosphatase. In the abdominal ultrasonographic evaluation with colored Doopler an anomalous vessel was visualized inserting in the caudal cava vein. There was a turbulent blood flow in the region of the anomalous vessel and dilation of the hepatic artery. This was compatible with a congenital extra-hepatic PSD. Doppler echocardiography was also performed to investigate cardiac abnormalities, but no changes were observed.

For better identification and delimitation of the anomalous vessel, it was chosen to perform a computed tomography scan. Pre-anesthesia was conducted with intramuscular administration of tramadol $\left(2 \mathrm{mg} \mathrm{kg}^{-1}\right)^{1}$ and acepromazine $\left(0.02 \mathrm{mg} \mathrm{kg}^{-1}\right)^{2}$. Anesthetic induction was performed with intravenous propofol ${ }^{1}$ dose effect, with subsequent orotracheal intubation and maintenance with isoflurane ${ }^{1}$ volatilized with oxygen using a universal vaporizer in a semi-closed system (Rees-Baraka).

Pre- and post-contrast computed tomography of the abdomen was performed in $2 \mathrm{~mm}$ and $1 \mathrm{~mm}$ pitch cross-sections and multiplanar reconstructions were made. Post-contrast phase revealed the anomalous vessel, with a tortuous path, passing in the hepatic hilar region ( $1.1 \mathrm{~cm}$ of diameter). It was originated from the cranial mesenteric vein and it was insert in the cranial margin of the caudal cava vein, in the topography of the branching of the left and right hepatic veins (Figure $1 \mathrm{~A}$ and $1 \mathrm{~B})$. The splenic, pancreato-duodenal, and gastro-duodenal veins were inserted into the anomalous vessel that deviated the portal fluid from the liver to the cranial margin of the caudal cava vein.

After the diagnosis, medical support treatment was started with intravenously maintenance fluid therapy (Ringer's solution, $3 \mathrm{~mL} / \mathrm{kg} / \mathrm{h}$ ) for three days, metronidazole $\left(15 \mathrm{mg} \mathrm{kg}^{-1}, \mathrm{BID}\right)^{3}$, intravenously, lactulose $(8 \mathrm{~mL} \mathrm{TID})^{4}$, orally, probiotic $(1 \mathrm{~g} \mathrm{BID})^{2}$, orally and hepatic $\operatorname{diet}^{5}$, for 15 days.

After clinical treatment, the surgery was performed. In the anesthetic protocol for the surgical procedure, $0.5 \mathrm{mg} \mathrm{kg}^{-1}$ of morphine ${ }^{1}$ was used, and after $15 \mathrm{~min}$, induction with propofol ${ }^{1}$ dose effect was performed, followed by intubation with orotracheal tube and isoflurane ${ }^{1}$ maintenance, with a universal vaporizer in the semi-closed system. With the animal positioned in dorsal decubitus, a trichotomy and antisepsis were performed in the entire ventral region of the abdomen and thorax. The abdominal incision was performed in the ventral midline from the caudal xiphoid cartilage to the beginning of the hypogastric 


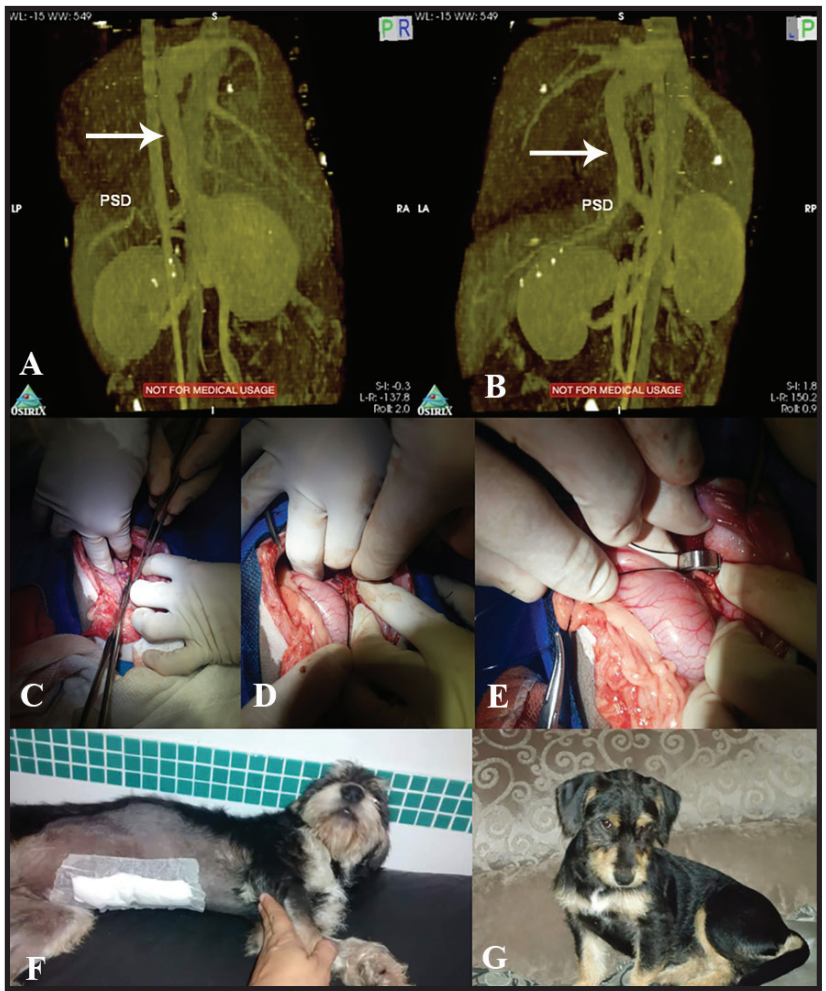

Figure 1. Congenital extra-hepatic portosystemic deviation in a mixedbreed dog. A- and B- Liver's post contrast computed tomography in $2 \mathrm{~mm}$ and $1 \mathrm{~mm}$ pitch cross sections and multiplanar reconstructions. In the post-contrast phase, an anomalous blood vessel of tortuous trajectory was observed, passing in the hepatic hilar region; calibrous $(1.1 \mathrm{~cm})$, having its origin in the cranial mesenteric vein and its insertion in the cranial margin of the caudal cava vein, in topography of branching of the left and right hepatic veins. C- The anomalous vessel was delicately dissected. D- The vessel was anchored with 2-0 nylon wires. E- Placement of the $5 \mathrm{~mm}$ ameroid constrictor ring. F- Patient bandaging about 2 days after surgery. G- Patient after three years demonstrating weight gain.

region. Guelpi retractors were positioned to facilitate abdominal exposure. The duodenum was retracted to the left and ventrally, facilitating the visualization of the caudal cava vein and its tributaries. It was observed the presence of an anomalous vessel, originating in the cranial mesenteric vein and entering the caudal cava vein, immediately after the renal veins reached the cava vein. The anomalous vessel was delicately dissected (Figure 1C) and anchored with 2-0 nylon yarn (Figure 1D), allowing placement of the $5 \mathrm{~mm}$ ameroid constrictor ring (Figure 1E). After placement of the implant the intestine was observed for some minutes to identify some sign of congestion. As no change in bowel staining occurred, the duodenum was repositioned, and the abdomen sutured with 2-0 nylon in cross-mattress pattern, the subcutaneous closure was performed with 3-0 nylon cushing pattern and the skin sutures were placed with 3-0 nylon in a horizontal separated mattress pattern (Wolff).
For the postoperative period, local dressings were performed every 2 days (Figure $1 \mathrm{~F}$ ). Analgesia was performed orally with tramadol ( $3 \mathrm{mg} \mathrm{kg}^{-1}$, TID $)^{1}$, for 5 days, meloxicam $\left(0.1 \mathrm{mg} \mathrm{kg}^{-1}, \mathrm{SID}\right)^{6}$, for 4 days and, dipyrone $\left(25 \mathrm{mg} \mathrm{kg}^{-1}, \mathrm{BID}\right)^{8}$, for 3 days. Oral amoxicillin (22 $\left.\mathrm{mg} \mathrm{kg}^{-1}, \mathrm{BID}\right)^{7}$, was administered for 10 days. The dog was kept on lactulose, for 7 days, probiotic $^{2}$ and hepatic diet ${ }^{5}$ for more 20 days.

The patient recovered completely from the surgery and after 20 days she was fed with puppy's diet, with no clinical signs of PSD. After 30 days of surgery, ultrasound control was performed, and it was possible to visualize the ameroid constrictor ring in the left cranial abdominal region, occluding the PSD close to its insertion to the caudal cava vein. The patient was followed up for more than 3 years, gained weight and showed no clinical signs of the disease, with a healthy normal life (Figure 1G).

\section{DISCUSSION}

This paper reports the occurrence of a congenital, extra-hepatic, solitary PSD, in a 7-month-old, mixed-breed spayed female dog. Although this pathology is more often diagnosed in pure-breed dogs $[4,8,15]$, clinical signs and the age of onset (7 months) were consistent with the suspicion of this pathology.

In dogs affected with PSD, there is a predominance of neurological, gastrointestinal and urinary disorders [1-3]. About $75 \%$ of PSDs presents clinical signs with less than one year of age, although some might present it until 10 years of age $[2,8,10,11]$. The affected young animals are usually evaluated overdue underdevelopment, reduced body height or weight loss due to poor digestion $[5,10]$, as evidenced in this report. There are usually signs of hepatic encephalopathy, which include ataxia, epileptic seizures and syncope, since the undigested protein is metabolized by intestinal (anaerobic and gram-negative) bacteria, resulting in the production of ammonia and several other toxins, which escapes hepatic metabolism, and once they remain liposoluble, they are able to cross-pass the blood-brain barrier and produce the neurological signs [2,5]. Likewise observed in this case there is an increased chance of neurological signs immediately after feeding, once the substrates will be more available for bacteria metabolism and synthesis of ammonia [5,11]. Nevertheless, the neurological signs presented by the dog in this report were mild. Diarrhea and vomiting may also occur due to poor digestion $[2,11]$. In the urinary tract, there might 
be development of uroliths, due to increased crystals, especially ammonium biurates, and the affected dogs with polyuria and polydipsia may present isostenuria or hyposostenuria [10]. However, gastrointestinal and urinary disorders were not evidenced in this report.

Haematological abnormalities and serum biochemistry of the reported case confirm the descriptions of the PSD in literature, with the occurrence of regenerative anaemia due to the failure of the liver to metabolize the red blood cells, as well as the lower absorption of nutrients by the organism [1]. Hypoalbuminemia and elevation of hepatic alanine aminotransferase (ALT) and alkaline phosphatase (AL) enzymes were also observed, which can be increased two to three times $[1,2,6]$.

Hypoglycemia is particularly frequent in dogs with extrahepatic congenital PSD [2,6], which may present hypocholesterolemia and reduction in urea levels [1], however, these abnormalities were not demonstrated by the patient in this report.

Bile acid concentrations should also be evaluated when PSD is suspected, especially for intra-hepatitc PSD, once its diagnosis may be harder, but this was not performed, once the anomalous vessel was clearly evident in the abdominal ultrasound. In the fasted animal, bile acid concentration may be normal or decreased once the liver removes bile acids from the systemic circulation during prolonged fasting. Nevertheless, post-stimulation concentration may be abnormally high in patients with PSD, due to escape of these substances from liver reabsorption $[1,6]$. The ammonia tolerance test is also an important indicator for PSD diagnosis. Ammonia, originated primarily through the catabolism of dietary amino acid, is metabolized and converted into urea in the liver. In cases of PSD, this biotransformation is compromised, which explains the existence of hyperammonemia, which can lead to hepatic encephalopathy $[2,6]$. However, this test was also not performed, since the diagnosis was obtained by ultrasonography and computed tomography $[12,14]$.

Exploratory abdominal radiographs are important as a screening test for PSD, to evaluate the possibility of microhepatia [9] however, in this study, ultrasound was primarily chosen because of its wide availability, non-invasiveness, and great assistance in abdominal investigation $[10,14]$. The use of color Doppler facilitate the identification of the vessel and characterization of the turbulent blood flow $[12,14]$, as evidenced in this case, although it was not possible to determine the precise location of the anomalous vessel.

Nuclear scintilography is a rapid, non-invasive method of documenting abnormal liver blood flow; however, advanced imaging techniques such as computed tomography (CT), have proved to be valuable for the diagnosis of vascular diseases associated to the liver, and in this case it proved decisive for identification and characterization of the anomalous vessel [12]. Three-dimensional reconstructions have improved understanding of anatomy and surgical planning and may aid in identification of concomitant vascular abnormalities [11,12,14].

Before submitting the animal to the surgical procedure, attention should be given to the patient's general clinical condition [9-11]. Medical support should include fluid therapy, normalization of acid-base disorders, and potassium supplementation if necessary $[2,11]$. It should also be instituted a highly digestible diet, fractionated several times a day, in which the main source of calories should be carbohydrates $[2,10]$. The manufactured diets, Hill's LD ${ }^{\circledR}$, and Royal Canin Hepatic ${ }^{\circledR}$, as used in this case, are a good options, but should also be supplemented with high quality protein such as cottage cheese or chicken $[2,10,11]$ which was not prescribed in these case, once the neurological signs were mild and several authors recommended protein restriction to reduce the substrate available to intestinal bacteria, responsible for ammonia production and hepatic encephalopathy $[2,5]$. The use of antibiotics is recommended in the management of PSD, especially those with action on anaerobic and gram-negative bacteria, related to ammonia production [5,11]. The antibacterials of choice are metronidazole, amoxicillin and neomycin sulfate $[2,8,11]$. Likewise, metronidazole was elected in these case and amoxicillin was used in the postoperative period.

Probiotics have been used to control hyperammonemia in human patients $[2,5,13]$ and were used in the patient of this study. Bacteria, such as Bifidobacterium bifidum and Streptococcus faecium, competitively decrease the urease-producing microbiota, reducing ammonia synthesis and absorption, although the concomitant use of antibiotics may compromise survival of probiotic's bacteria $[5,11,13]$. The use of lactulose, as performed, but also fructooligosaccharides (FOS), has synergistic action, in stimulating the growth of beneficial bacteria [5]. 
Dogs with congenital PSD may also exhibit hepatic oxidative damage, and therefore the use of antioxidants may help to protect the hepatocyte membrane, especially d-alpha-tecoferol, S-adenosyl-L-methionine, and silybin. Ursodeoxycholic acid may also help in preventing biliary stasis and subsequent inflammation of bile ducts [2,3]. These drugs were not used and were also not necessary in the reported case.

According to the literature the surgical technique of choice for the single congenital PSD, is the placement of the ameroid constrictor ring [7,10,11]. This implant consists in a stainless-steel ring with a groove inside it, with a layer of hygroscopic casein material that expands when in contact with the interstitial fluid, promoting the gradual occlusion of the anomalous vessel, as shown in this case, by ultrasound, 30 days after surgery [7]. Alternatively, cellophane bands may be used, which induce an initial acute inflammatory response, followed by a chronic inflammatory low-grade tissue reaction, occluding the anomalous vessel [7,10]. Post-surgical morbidity is associated with hypoglycaemia and the mortality rate varies from 5 to $21 \%$ in the postoperative period $[1,11]$. Death may occur during or shortly after the surgical procedure and is caused by portal hypertension, anesthetic complications, or epileptic status [9]. However, no complications were observed in the trans and postoperative period. When the surgery is successful the clinical improvement occurs on the first postoperative day [10]. Hepatic function recovery occurs between 2 to 4 months after surgery and clinical recovery of the animal is complete, as evidenced in this study $[10,11,15]$.

\section{CONCLUSION}

Diagnosis of PSD can be easily accomplished by an experienced ultrasonographer, although computed tomography is highly recommended for surgical planning, once it allows identification and characterization of the anomalous vessel. Medical treatment was effective to support this patient until surgery. The application of the ameroid constrictor ring produced an excellent result in the gradual occlusion of the anomalous vessel, thereby preventing portal hypertension and promoting the complete clinical recovery of the dog with a solitary congenital extra-hepatic PSD. The patient of this report did not present any complications in the postoperative period and for more than three years, showing total remission of the clinical signs and good life's quality.

\section{MANUFACTURERS}

${ }^{1}$ Cristália Produtos Químicos Farmacêuticos Ltda. Itapira, SP, Brazil. ${ }^{2}$ Vetnil Ind. e Com. de Produtos Veterinarios Ltda. Louveira, SP, Brazil. ${ }^{3}$ Isofarma industrial Farmacêutica Ltda. Precabura Eusebio, CE, Brazil.

${ }^{4}$ Daiichi Sankyo Brasil Farmacêutica Ltda. Barueri, SP, Brazil.

${ }^{5}$ Royal Canin do Brasil. Descalvado, SP, Brazil.

${ }^{6}$ Ouro Fino Saúde Animal Ltda. Cravinhos, SP, Brazil.

${ }^{7}$ GlaxoSmithKline Brasil Ltda. Rio de Janeiro, RJ, Brazil.

${ }^{8}$ Hipolabor Farmacêutica Ltda. Sabará, MG, Brazil.

Declaration of interest. The authors report no conflicts of interest. The authors alone are responsible for the content and writing of paper.

\section{REFERENCES}

1 Center S.A. 1997. Fisiopatologia, diagnóstico laboratorial e afecções do fígado. In: Ettinger S.J. \& Feldman E.C. (Eds). Tratado de Medicina Interna Veterinária: Moléstias do Cão e do Gato. 4.ed. São Paulo: Manole, pp.1745-1816.

2 Cornelius L.M. 1996. Enfermidades Hepáticas. In: Lorez M.D., Cornelius L.M. \& Ferguson D.C. (Eds). Terapêutica Clínica em Pequenos Animais. Rio de Janeiro: Interlivros, pp.200-216.

3 Johnson S.E. 2004. Hepatopatias crônicas. In: Ettinger S.J. \& Feldman E.C. (Eds). Tratado de Medicina Interna Veterinária: Doenças do Cão e do Gato. 5.ed. Rio de Janeiro: Guanabara Koogan, pp.1369-1397.

4 Kyles A.E., Hardie E.M., Mehi M. \& Gregory C.R. 2002. Evaluation of ameroid ring constrictors for the management of single extrahepatic portosystemic shunts in cats. Journal of the American Veterinary Medical Association. 220: 1341-1347.

5 Loguercio C., Abbiati R., Rinaldi M., Romano A., Del Vecchio Bianco C. \& Coltori M. 1995. Long term effects of Enterococcus faeciumSF 68 versus lactulose in the treatment of pacients with cirrhosis and grade I-II hepatic encephalopathy. Journal of Hepatology. 23(1): 39-46.

6 Meyer D.J., Coles E.H. \& Rich L.J. 1995. Anormalidades em testes hepáticos. In: Medicina de laboratório veterinária: Interpretação e diagnóstico. São Paulo: Roca, pp.47-61.

7 Murphy S.T. 2001. A Comparison of the Ameroid Constrictor Versus Ligation in the Surgical Management of Single Extrahepatic Portosystemic Shunts. Journal of the American animal Hospital Association. 37: 390-396. 
8 Reginatto R.C., Frehse M.S., Tanaka N.M., Fávero V., Sprea G., Bach F.S. \& Sanson M.N. 2011. Shunt portossistêmico extra-hepático em cadela maltês de 8 meses. Ciências Agrárias. 32(2): 739-746.

9 Ricciardi M. 2016. Splenophrenic portosystemic shunt in dogs with and without portal hypertension: can acquired and congenital porto-caval connections coexist? Open Veterinary Journal. 6(3): 185-193.

10 Santos R.O., Sanchez C.A., Rocha R.C., Mello M.E. \& Carvalho A.R. 2014. Shunt portossistêmico em pequenos animais. PUBVET. 8(18): 1-17.

11 Sassaki R.A., Sassaki E.L. \& Sassaki M.S. 2001. Desvio porto-sistêmico congênito simples extra hepático: correção com constritor ameróide. Relato de caso. Clínica Veterinária. 33: 27-32.

12 Schaub S., Hartmann A., Schwarz T., Kemper K., Pueckler K.H. \& Schneder M.A. 2016. Comparison of contrastenhanced multidetector computed tomography angiography and splenoportography for the evaluation of portosystemicshunt occlusion after cellophane banding in dogs. BMC Veterinary Research. 12: 283.

13 Schumann C. 2002. Medical, nutritional and technological properties of lactulose. An update. European Journal of Nutrition. 41(1): 17-25.

14 Silva V.C., Mamprim M.J., Vulcano L.C. \& Doiche D.P. 2009. Ultrassonografia doppler e angiografia tomográfica computadorizada no diagnóstico de desvios portossistêmicos. Revisão de Literatura. Clínica Veterinária. $78:$ 70-78.

15 Tobias K.M. 2003. Determination of inheritance of single congenital portosystemic shunts in Yorkshire Terriers. Journal of the American Animal Hospital Association. 39: 385-389. 